\title{
ADSORÇÃO DO CORANTE AZUL DE METILENO EM PARTÍCULAS DE ARGILOMINERAIS: ANÁLISE DOS TAMANHOS DAS PARTÍCULAS
}

\author{
Rogerio Antonio Sartori, Luis Carlos de Morais, Nelson Consolin-Filho*\# e Délcio Dias Marques \\ Departamento de Química, Universidade Federal do Acre, 69915-900 Rio Branco - AC, Brasil \\ Fergus Gessner \\ Instituto de Química de São Carlos, Universidade de São Paulo, CP 780, 13560-970 São Carlos - SP, Brasil
}

Recebido em 15/4/10; aceito em 4/11/10; publicado na web em 18/2/11

\begin{abstract}
ADSORPTION OF METHYLENE BLUE ON CLAY MINERALS PARTICLES: ANALYSIS OF THE CLAY PARTICLE SIZES. Interactions of cationic dye methylene blue (MB) with clay particles in aqueous suspension have been extensively studied. As already known, the number of natural negative charges on the clay modifies significantly the particle sizes dispersed in water and therefore the nature of the interaction with the dye. This work evaluated with UV-Vis spectroscopy method how the clay particle sizes weighted on the adsorption and rearrangement of the dye molecules in aqueous system. The results obtained from light-scattering measurements confirmed that larger particles are found in suspensions containing the high-charged clays as the visible absorption band related to the MB aggregates $(570 \mathrm{~nm})$ on these suspensions prevailed.
\end{abstract}

Keywords: clays; methylene blue; UV-Vis spectroscopy.

\section{INTRODUÇÃO}

Sempre houve um interesse muito grande nos processos de adsorção de moléculas orgânicas em sistemas coloidais aquosos, sobretudo naqueles contendo argilominerais. ${ }^{1-12} \mathrm{O}$ conhecimento sobre tais processos manifesta-se em diferentes setores da sociedade como agricultura, indústrias, águas, etc.. ${ }^{13}$ Diversas espécies químicas podem ser adsorvidas em diferentes substratos, como as argilas.

\section{$\operatorname{Argilas}^{13-17}$}

Os argilominerais são os minerais constituintes das argilas. Eles são silicatos hidratados dispostos em camadas (ou lamelas) formadas por subcamadas (ou "folhas") de tetraedros de silício (ou alumínio) e oxigênio e subcamadas de octaedros compostos por átomos de alumínio (ou ferro e magnésio) e oxigênio. Há uma classificação dos argilominerais em função da disposição das subcamadas, a qual considera a razão entre as subcamadas tetraédrica e octaédrica, respectivamente. Por exemplo, um mineral formado por uma subcamada tetraédrica e uma octaédrica é classificado como argilomineral do tipo 1:1. Se houver duas subcamadas tetraédricas e uma octaédrica, o material é classificado como mineral do tipo 2:1.

Pode ainda haver substituições de alguns dos átomos nas estruturas que compõem o argilomineral. Quando átomos de $\mathrm{Si}^{4+}$ são substituídos por $\mathrm{Al}^{3+}$ na folha octaédrica, e átomos de $\mathrm{Al}^{3+}$ são substituídos por $\mathrm{Mg}^{2+}$, o material fica com carga líquida negativa, que é contrabalanceada por íons metálicos como $\mathrm{Na}^{+}, \mathrm{K}^{+}, \mathrm{Ca}^{2+}$, etc., ou íons moleculares como $\mathrm{NH}_{4}^{+}$, moléculas de surfactantes e corantes catiônicos, etc., presentes nas superfícies das partículas. A quantidade desses contra-íons pode ser medida e resulta na capacidade de troca catiônica (ctc) da argila, expressa usualmente em miliequivalentes do

*e-mail: nelconsolin@ hotmail.com

\#Depto. de Química, Universidade Tecnológica Federal do Paraná, Campo

Mourão - PR, Brasil íon para cada $100 \mathrm{~g}$ de argila (meq/100 g). As montmorilonitas, por exemplo, possuem ctc que podem variar de 70 até $170 \mathrm{meq} / 100 \mathrm{~g} .{ }^{13}$

Outra característica de algumas argilas é a sua capacidade de adsorção de moléculas de água na superfície das camadas, fazendo com que haja um inchamento e provocando a dispersão das mesmas, quando há água suficiente para isso. No entanto, geralmente essa dispersão não é total e na suspensão ainda permanecem algumas partículas associadas face-a-face, chamadas de tactoides. $\mathrm{O}$ tamanho dos tactoides, por sua vez, está relacionado diretamente com a quantidade de cargas negativas que a argila possui, entre outros fatores. Segundo Norrish, a energia de coesão por metro quadrado de área interlamelar, $E_{i},{ }^{18}$ é dada por

$$
E_{i}=k \sigma_{i}\left(1 / D_{1}-1 / D_{2}\right)
$$

onde $\sigma_{i}$ é a densidade de cátions $i\left(\right.$ cargas $\left.\mathrm{m}^{-2}\right)$; $k$ é um fator que depende do meio e da carga do cátion; $D_{1}$ e $D_{2}$ são as distâncias entre o cátion e cada uma das lamelas adjacentes.

A Equação 1 mostra que quando a densidade de carga aumenta, a energia que mantém as lamelas unidas também aumenta, tornando a dispersão das camadas mais difícil e, consequentemente, resulta em tactoides de tamanhos maiores na suspensão. Ou seja, certa argila com ctc relativamente alta tende a formar suspensões com partículas maiores do que outra argila com ctc mais baixa.

\section{Corantes catiônicos}

Moléculas de corantes catiônicos exibem um comportamento característico em função de sua concentração em solução. Elas podem se unir formando dímeros moleculares, ou mesmo trímeros e agregados maiores. ${ }^{19-21}$ Mesmo em concentrações bem baixas, da ordem de $10^{-4}$ mol L ${ }^{-1}$, muitas soluções de corantes já apresentam a presença de agregados. Os espectros de absorção de luz visível são diferentes para as moléculas que se encontram nas formas agregadas daqueles vistos para os monômeros do corante (i.e., moléculas que se encontram separadas 
umas das outras), uma vez que a agregação altera significativamente os níveis de energia para as transições eletrônicas das mesmas. ${ }^{19,22,23}$

Como exemplo, em soluções de azul de metileno, AM, com concentrações da ordem de $10^{-6} \mathrm{~mol} \mathrm{~L} \mathrm{~L}^{-1}$, nota-se uma banda de absorção máxima em $670 \mathrm{~nm}$ correspondente aos monômeros de AM. Em concentrações mais altas, a banda dos monômeros diminui e outra banda com máximo em 605 nm, atribuída aos dímeros de AM, passa a predominar. Em concentrações ainda maiores é possível observar outra banda por volta de $575 \mathrm{~nm}$, referente aos trímeros e agregados maiores..$^{22,23}$

Em meio aquoso altamente ácido o AM exibe também uma banda com máximo em 765 nm, que é atribuída aos monômeros protonados do corante.

\section{Corantes em $\operatorname{argilas}^{22-30}$}

Devido principalmente ao fato dos corantes catiônicos exibirem essas variações espectrais em função de sua concentração ou do meio em que se encontram, é que eles se constituem boas sondas para avaliação de sistemas chamados micro-heterogêneos, como são as argilas em suspensões. As informações acerca dos sítios de adsorção das argilas que são obtidas em meio aquoso podem diferir significativamente daquelas realizadas para sistemas no estado sólido. O sistema em água é um sistema dinâmico e possibilita a adsorção/dessorção de espécies em função do tempo. Diversos estudos mostraram que ocorre uma variação espectral de corantes adsorvidos em argilas no meio aquoso. ${ }^{24,25,30,31}$ Diversos autores propuseram alguns eventos prováveis que poderiam ocorrer, principalmente através de estudos de suspensões com a argila SWy-1, uma montmorilonita proveniente de Wyoming, Estados Unidos. Os resultados sugerem que no momento em que o corante é adicionado à suspensão de argila, ocorre inicialmente uma adsorção preferencial nas superfícies externas dos tactoides em suspensão. Pela proximidade das moléculas nestes sítios, ocorre rapidamente um equilíbrio de agregação no referido ambiente. Conforme o tempo passa, ocorre uma reordenação das moléculas nas superfícies externas levando à formação de monômeros do corante que, por sua vez, migram para os espaços interlamelares (superfícies internas) onde sofrem protonação pelas moléculas de água que se encontram no local..$^{32} \mathrm{Em}$ alguns casos, alguns monômeros que estejam na solução podem migrar diretamente para esses espaços internos, sem adsorver inicialmente nas superfícies externas.

Em tempos maiores, as partículas tendem a se aglomerar, ocorrendo então sua floculação. Esse fenômeno faz com que novos domínios interlamelares sejam criados, podendo, neste caso, aumentar ainda mais a quantidade de AM protonado.

Muitos desses processos são alterados quando a argila empregada possui alta ou baixa ctc. Esta propriedade, como já exposto, é um dos principais fatores que alteram os tamanhos das partículas em suspensão. Dessa forma, este trabalho avaliou qual é a influência do tamanho das partículas de argila nos mecanismos de adsorção/rearranjo de moléculas de corante em meio aquoso, por meio do estudo das bandas de absorção eletrônica do AM em argilas com diferentes quantidades de carga lamelar.

\section{PARTE EXPERIMENTAL}

Foi utilizado o corante azul de metileno proveniente da Riedel-deHaën, sem tratamentos prévios. As montmorilonitas foram obtidas da Source Clays, da Clay Minerals Society, Universidade do Missouri, EUA e a hectorita foi originária da Laporte Industries, Reino Unido. Todas as amostras foram purificadas e transformadas na forma sódica, de acordo com procedimento já estabelecido na literatura. A Tabela 1 mostra algumas propriedades das argilas empregadas neste trabalho.

Os experimentos de espalhamento de luz por laser $(532 \mathrm{~nm})$ foram feitos com um goniômetro modelo BI-200M da Brookhaven Intruments Corp. para a determinação da distribuição multimodal dos tamanhos das partículas das argilas em suspensão aquosa. Nestes experimentos, foi utilizada água pré-filtrada em sistema Millipore Milli-Q para o preparo das suspensões e, a fim de remover outras impurezas, foram feitas filtrações adicionais em membrana de $0,45 \mu \mathrm{m}$, pouco antes da realização das medidas. Um banho termostático de circulação manteve a temperatura constante em $25^{\circ} \mathrm{C}$ na célula da amostra e as medidas foram obtidas em ângulo de espalhamento de $90^{\circ}$.

Os espectros de absorção eletrônica foram obtidos com um espectrofotômetro Hitachi U-2000 acoplado a um computador IBM. A faixa de varredura no espectro visível se estendeu entre 400 e $800 \mathrm{~nm}$.

\section{RESULTADOS E DISCUSSÃO}

\section{Tamanhos de partículas}

A Figura 1 mostra as diferentes distribuições multimodais de tamanho para as argilas LapRD, SWy-1, SAz-1 e SCa-3. Como pode ser visto na Figura 1a, a suspensão de laponita é a que apresenta menor distribuição de tamanho de todas as outras suspensões. Pelo fato da LapRD conhecidamente produzir suspensões muito dispersas, as suas partículas são muito pequenas e espalham pouca luz. Isso exige cuidados adicionais no momento em que se prepara sua suspensão, principalmente durante a filtração da água usada. Percebe-se ainda nas suspensões das outras argilas que não existe uma única distribuição de tamanho, mas diferentes modos de distribuição, isso porque geralmente suspensões de argila apresentam uma alta polidispersividade.

Tabela 1. Propriedades e composição das argilas utilizadas ${ }^{34,35}$

\begin{tabular}{|c|c|c|c|c|}
\hline Propriedades & SWy-1 & SAz-1 & $\mathrm{SCa}-3$ & LapRD \\
\hline $\operatorname{ctc}(\mathrm{meq} / 100 \mathrm{~g})$ & 76,4 & 120 & 153 & 55 \\
\hline Área específica $\left(\mathrm{m}^{2} \mathrm{~g}^{-1}\right)$ - BET & 31,82 & 97,42 & 82,5 & 370 \\
\hline Tipo & Montmorilonita natural & Montmorilonita natural & Montmorilonita natural & hectorita sintética \\
\hline Origem & Wyoming, EUA & Arizona, EUA & Califórnia, EUA & Laporte Inds. \\
\hline \multicolumn{5}{|l|}{ Composição } \\
\hline$\% \mathrm{SiO}_{2}$ & 62,9 & 60,4 & 52,8 & 66,03 \\
\hline$\% \mathrm{Al}_{2} \mathrm{O}_{3}$ & 19,6 & 17,6 & 15,7 & 0,30 \\
\hline$\% \mathrm{Fe}_{2} \mathrm{O}_{3}$ & 3,35 & 1,42 & 1,06 & 0,06 \\
\hline$\% \mathrm{MgO}$ & 3,05 & 6,46 & 7,98 & 29,03 \\
\hline$\% \mathrm{CaO}$ & 1,68 & 2,82 & 0,95 & 0,34 \\
\hline$\% \mathrm{FeO}$ & 0,32 & 0,08 & $<0,10$ & ND \\
\hline
\end{tabular}


Para a SWy-1, Figura 1b, aparece uma distribuição principal, com intensidade máxima relativamente maior do que a da LapRD.

LapRD $0,25 \mathrm{~g} \mathrm{~L}^{-1}$

a)

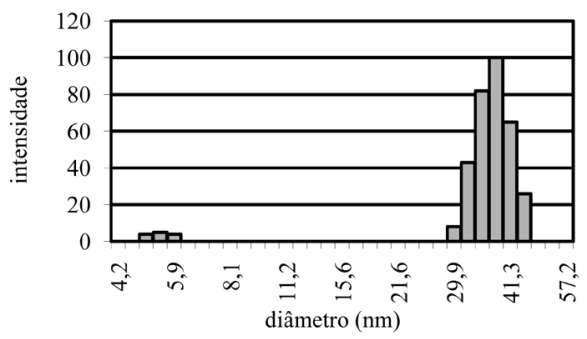

SWy-1 0,25 g L-1

b)

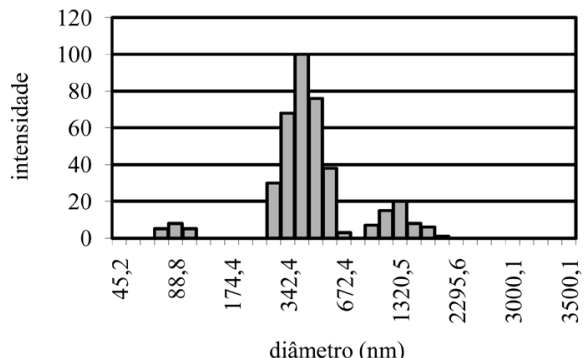

SAz-1 $0,25 \mathrm{~g} \mathrm{~L}^{-1}$

c)

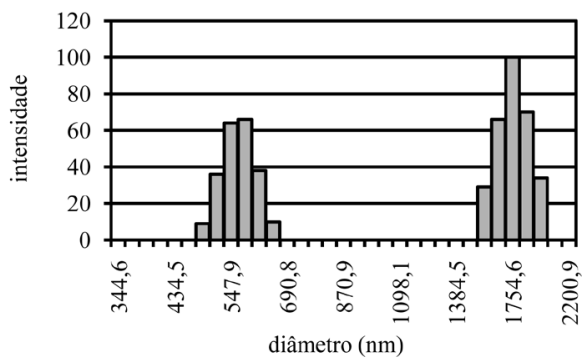

Figura 1. Distribuições multimodais de tamanho de suspensões de (a) LapRD, (b) SWy-1 e (c) SAz-1, todas 0,25 g $L^{-1}$

Na suspensão de SAz-1, Figura 1c, verificam-se duas distribuições principais em regiões maiores do que as da LapRD e SWy-1 e ambas são muito semelhantes àquelas encontradas também para a SCa-3.

No caso de argilas um importante fator a ser considerado se refere à estabilidade das suspensões. Com o passar do tempo, as partículas tendem a se agregar, formando aglomerados maiores que, por sua vez, podem modificar as medidas de espalhamento por laser. Por isso, essa técnica pode ser usada também para se determinar estabilidade de suspensões, com ou sem a presença de outras espécies, como eletrólitos e moléculas orgânicas, como corantes. Mas nesse caso, todas as medidas foram realizadas logo após o preparo das suspensões e os resultados sempre foram razoavelmente reprodutíveis. Em alguns casos, a floculação das partículas interferiu nos resultados, obtendo-se valores diferentes (partículas maiores).

Quando a luz, de modo geral, incide sobre pequenas partículas, ela é espalhada em todas as direções (espalhamento de Rayleigh), contanto que as partículas sejam pequenas quando comparadas ao comprimento da onda da luz incidente (abaixo de $250 \mathrm{~nm}$ ). Se a luz é um laser, logo é monocromática, então se observa uma flutuação dependente do tempo na intensidade do espalhamento. Isso se deve essencialmente ao movimento browniano das partículas na solução.
Logo, as distâncias entre as partículas espalhadoras de luz mudam constantemente com o tempo e isso pode provocar diversas interferências, construtivas ou destrutivas, na radiação da luz espalhada.

Dentre as argilas analisadas, a LapRD é a que apresenta tamanhos menores em suspensão, por isso a determinação da sua distribuição está sujeita a maiores flutuações. A SWy-1 apresentou um máximo muito próximo ao limite imposto pela luz incidente $(532 \mathrm{~nm}), \log 0$ também pode apresentar algumas interferências. Nas demais argilas, SAz-1 e SCa-3, os tamanhos sempre foram superiores ao do comprimento de onda do laser e, por isso, têm comportamento mais estável. Uma maneira de reduzir a interferência nos espalhamentos é utilizar concentrações mais baixas de argila nas suspensões, por isso se trabalhou sempre com suspensões de $0,25 \mathrm{~g} \mathrm{~L}^{-1}$. No entanto, é importante ressaltar que os tamanhos das partículas de argilas em suspensão, por sua vez, dependem significativamente de sua concentração. Em quantidades maiores, as partículas têm mais possibilidades de interações, favorecendo a agregação das mesmas. Em suspensões muito diluídas, predominam partículas com distribuições menores de tamanhos.

Também foram feitos experimentos com essas mesmas suspensões produzindo espalhamento de luz visível por meio de espectrofotômetro na faixa de 400 a $800 \mathrm{~nm}$. Os espectros originados devido ao espalhamento de luz das partículas nessa faixa de comprimento de onda são mostrados na Figura 2 (suspensão de argila contra água).

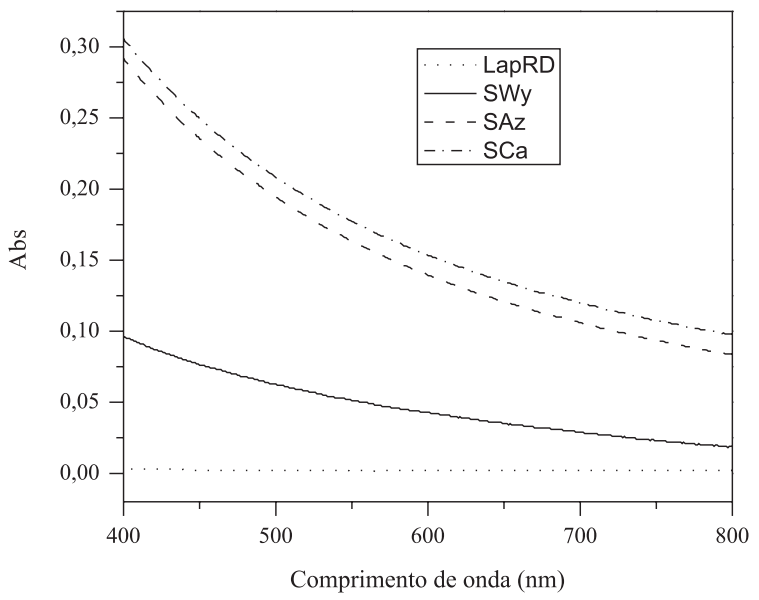

Figura 2. Espalhamento de luz como absorbância de suspensões contendo LapRD, SWy-1, SAz-1 e SCa-3, todas 0,25 g L

Como pode ser visto, há uma ordem crescente de tamanhos relativos de partículas: LapRD $<$ SWy-1 $<$ STx-1 $<$ SAz-1 $\cong \mathrm{SCa}-3$. Esses resultados estão em uma concordância muito boa com aqueles obtidos com o uso de espalhamento com laser, se forem tomados os tamanhos relativos às distribuições máximas de cada suspensão como parâmetro de comparação.

Valores de absorbância não necessariamente representam medidas de espalhamento de luz propriamente ditas. No entanto, Banin e Lahav ${ }^{36}$ mostraram ser possível estimar os números relativos de camadas de argilas associadas por tactoide em suspensão pela análise comparativa de espectros obtidos de forma similar aos mostrados na Figura 2, todos em relação à LapRD, considerada pelos autores como sendo a mais dispersa de todas.

Como o objetivo deste trabalho foi avaliar as variações espectrais do corante AM em suspensões aquosas de argilas com diferentes tamanhos de partículas, os experimentos de espalhamento de luz laser ou visível tiveram apenas a intenção de demonstrar qualitativamente as diferenças entre os tamanhos das partículas em suspensão das argilas empregadas. Eles não têm o compromisso de quantificar com exatidão os tamanhos das mesmas. Por isso, decidiu-se não aplicar métodos comumente usa- 
dos para caracterizar partículas coloidais de dimensões nanométricas ou submicrométricas como, por exemplo, a espectroscopia de correlação de fótons (espalhamento dinâmico de luz). ${ }^{37}$

\section{Azul de metileno em LapRD}

A Figura 3a mostra os espectros de AM em suspensão contendo LapRD em condições semelhantes àquelas vistas em outros trabalhos com a suspensão da argila SWy-1.23-25

a)

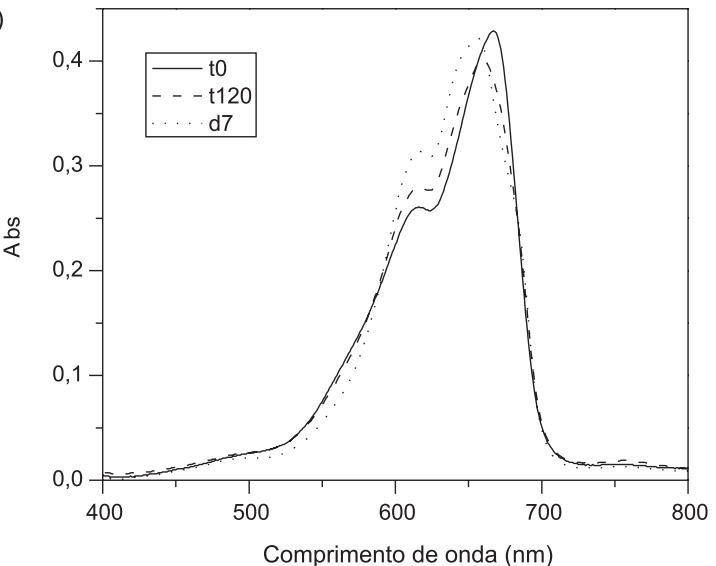

b)

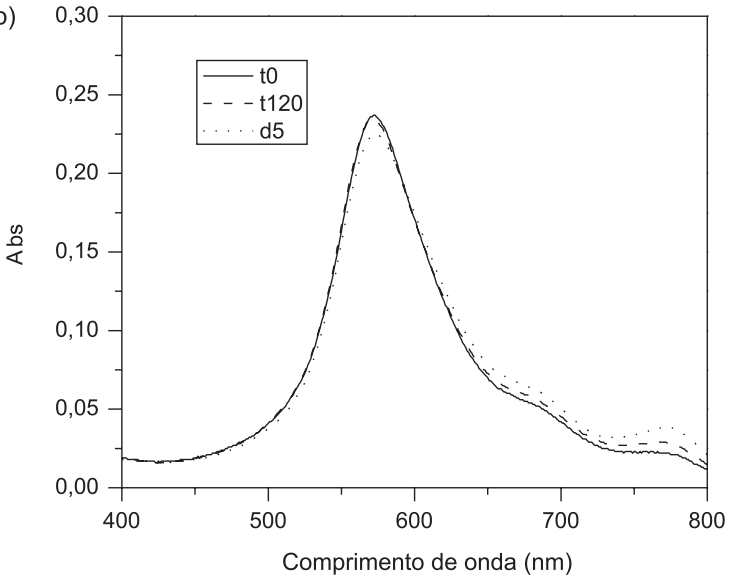

Figura 3. Espectros de AM 5,6 $\times 10^{-6} \mathrm{~mol} \mathrm{~L}^{-1}$ em suspensões de (a) LapRD, $e$ (b) SCa-3; [argila] $=0,064 \mathrm{~g} \mathrm{~L}^{-1}$. t estão expressos em minutos e d em dias

Nota-se que no momento da adição do corante na suspensão, apenas uma banda intensa em 668 nm é detectada. Essa banda é atribuída aos monômeros de AM adsorvidos na laponita. ${ }^{38}$ Como a LapRD se dispersa em grande extensão no meio aquoso, há um grande número relativo de partículas com área superficial suficiente para acomodar as moléculas de AM como monômeros. Isso distancia as moléculas umas das outras e a possibilidade de se agregarem é pequena (a área mencionada aqui não se refere àquela mencionada na Tabela 1 , a qual é obtida em condições totalmente diferentes, ou seja, em estado sólido, enquanto neste trabalho têm-se partículas dispersas em água com diferentes tamanhos e, consequentemente, diferentes áreas).

Em tempos médios e longos não se nota praticamente nenhuma variação espectral, mas apenas um ligeiro deslocamento da banda dos monômeros de AM para $656 \mathrm{~nm}$. Isso é explicado pela agregação das partículas de laponita em suspensão. Conforme se aglomeram, essas partículas "aprisionam" as moléculas do corante em novos ambientes internos que são formados. Esses espaços recém-criados, por sua vez, diferem dos espaços interlamelares que possuem sítios ácidos, conforme visto para a argila SWy- $1 .{ }^{38} \mathrm{Na}$ LapRD não é observada a banda dos monômeros protonados com intensidade significativa em uma ampla faixa de concentrações de argila e corante. Pelo fato dessa argila se dispersar em grande extensão, há poucas lamelas associadas e, por isso, poucos espaços com sítios ácidos.

\section{Azul de metileno em SAz-1 e SCa-3}

A Figura 3b mostra os espectros de AM para suspensão da argila SCa-3. Nota-se principalmente a presença da banda dos agregados de AM, desde os tempos mais curtos até os mais longos, para ambas as argilas. Resultados muito similares foram obtidos com estudos das suspensões da argila SAz-1.

As argilas SAz-1 e SCa-3 têm como característica a elevada densidade de carga (alta ctc). Como já citado, essa grande carga superficial dificulta a dispersão das partículas em água, uma vez que a energia de coesão entre as lamelas é grande, de acordo com a Equação de Norrish. Como resultado, tem-se uma suspensão contendo poucos tactoides e com tamanhos relativamente elevados, fato que foi constatado pelas medidas de espalhamento de luz.

Uma vez que poucas partículas estão dispersas no meio, há então pouca área superficial externa onde as moléculas de corante podem adsorver. Isso leva a um adensamento das moléculas e facilita sua agregação. Como nas partículas não há espaço suficiente para as moléculas se rearranjarem, não ocorrem os equilíbrios de desagregação que levariam à formação de monômeros de $\mathrm{AM}$, permanecendo grande parte das moléculas agregadas nas superfícies externas dos tactoides. Como a energia de coesão entre as camadas dessas argilas é grande o suficiente para mantê-las muito próximas umas das outras, a migração dos eventuais monômeros formados para os sítios ácidos interlamelares pode ficar fisicamente dificultado. ${ }^{31,39}$

Foram realizados experimentos em diversas concentrações de corantes e de argilas e, somente em determinadas condições, foi possível verificar a presença da banda dos monômeros protonados com redução da banda dos agregados de AM. Somente quando a razão de concentração [argila]/[AM] é mantida relativamente alta é que se observam os mesmos rearranjos constatados em SWy-1. Isso é explicado em função de que nessas condições há um grande número de sítios de adsorção (grande área superficial externa) em relação ao número de moléculas de AM em suspensão, o que possibilita ocorrerem equilíbrios de desagregação do corante nas superfícies externas dos tactoides, levando à formação de monômeros e, consecutivamente, de monômeros protonados.

A Figura 4 elucida esse fato, mostrando os espectros de AM 2,0 $\mathrm{x}$ $10^{-6} \mathrm{~mol} \mathrm{~L}^{-1} \mathrm{em}$ suspensões da argila SCa-3 em três diferentes concen-

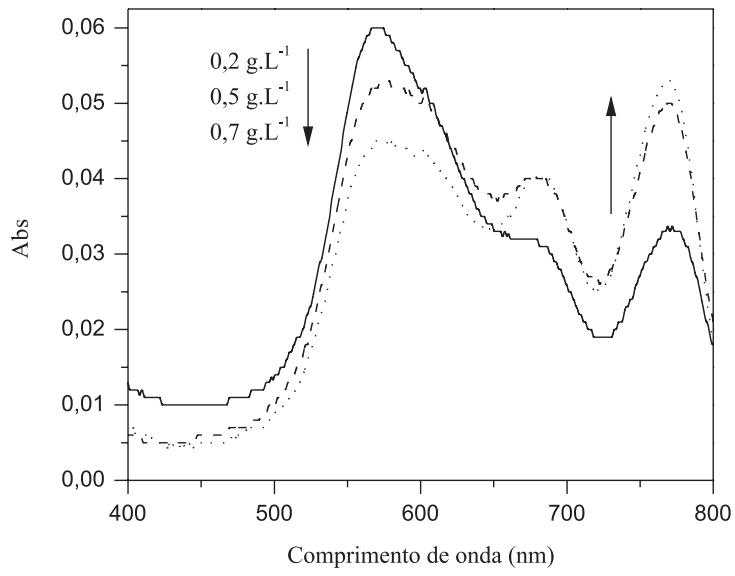

Figura 4. Espectros de AM 2,0 $\times 10^{-6} \mathrm{~mol} \mathrm{~L} L^{-1}$ em suspensões de SCa-3 com três concentrações: 0,$2 ; 0,5$ e 0,7 $\mathrm{g} \mathrm{L}^{-1,}$ após 30 dias 
trações, após 30 dias da adição do corante. Pode ser visto que conforme a concentração da argila aumenta na suspensão, os agregados diminuem e mais monômeros protonados são formados. Ainda assim, esses efeitos ocorrem em menor extensão do que aqueles observados com AM em SWy-1, restando ainda muitos agregados do corante adsorvidos, mesmo depois de 30 dias. Experimentos iguais foram repetidos com a argila SAz-1 e os resultados são similares aos obtidos com a SCa-3.

\section{CONCLUSÃO}

O uso de AM como sonda para investigação de sítios de adsorção das argilas estudadas foi possível devido ao seu comportamento espectral característico, uma vez que sua banda de absorção na região do visível é alterada significativamente em função dos microambientes formados pelas partículas de argila em suspensão.

Os tamanhos das partículas das argilas estudadas, por sua vez, são fator importante na forma como o AM pode ser adsorvido, e são função da quantidade de carga das argilas.

A laponita RD, por apresentar partículas pequenas em suspensão, possui uma grande área superficial para adsorção das moléculas de $\mathrm{AM}$, favorecendo a presença dos monômeros. A alta dispersão dessa argila produz poucos sítios ácidos interlamelares.

A SWy-1 também tem partículas pequenas em suspensão, no entanto, a presença de alguns tactoides diminui a superfície externa total para adsorção do corante, favorecendo alguns agregados inicialmente. Os rearranjos levam à formação de monômeros e monômeros protonados, devido à existência dos sítios ácidos nessa argila. Tanto a SAz-1 quanto a SCa-3 têm partículas com menor área externa, devido aos tamanhos maiores dos tactoides. Isso favorece a adsorção de agregados de AM. Os rearranjos não ocorrem em grande escala e a forte energia de coesão das camadas nessas argilas inibe fisicamente a migração dos monômeros de AM para os espaços interlamelares (mais estreitos), produzindo poucos monômeros protonados.

\section{AGRADECIMENTOS}

À Universidade Federal do Acre, ao Instituto de Química de São Carlos pela infraestrutura de trabalho, ao $\mathrm{CNPq}$ (proc. $\mathrm{n}^{\underline{0}}$ 302756/2009-4) e à CAPES pelos apoios financeiros.

\section{REFERÊNCIAS}

1. Alkaram, U. F.; Mukhlis, A. A.; Dujaili, A. H.; J. Hazard. Mater. 2009, $169,324$.

2. Juang, T. Y.; Chen, Y. C.; Tsai, C. C.; Dai, S. A.; Wu, T. M.; Jeng, R. J.; Appl. Clay Sci. 2010, 48, 103.

3. Delbem, M. F.; Valera, T. S.; Valenzuela-Díaz, F. R.; Demarquette, N. R.; Quim. Nova 2010, 33, 309.

4. Paiva, L. B.; Morales, A. R.; Valenzuela-Díaz, F. R.; Appl. Clay Sci. 2008, $42,8$.

5. Eren, E.; Afsin, B.; Dyes Pigm. 2008, 76, 220.

6. Weng, C. H.; Pan, Y. F.; J. Hazard. Mater. 2007, 144, 355.
7. Zaia, D. A. M.; Zaia, C. T. B. V.; Quim. Nova 2006, 29, 786.

8. Bayrak, Y.; Microporous Mesopouros Mater. 2006, 87, 203.

9. Cavalcante, A. M.; Torres, L. G.; Coelho, G. L. V.; Braz. J. Chem. Eng. 2005, 22, 75 .

10. Hsu, Y. H.; Wang, M. K.; Pai, C. W.; Wang, Y. S.; Appl. Clay Sci. 2000, 16, 147.

11. Cox, L.; Hermosín, M. C.; Cornejo, J.; Clay Miner. 1994, $29,767$.

12. Stul, M.; Uytterhoeven, J. B.; J. Chem. Soc. Faraday I 1975, 71, 1396.

13. Santos, P. S.; Ciência e tecnologia de argilas, $2^{\mathrm{a}}$ ed., Edgard Blücher: São Paulo, 1992, V1-3.

14. Gomes, C. F.; Argilas - o que são e para que servem, Fundação CaloustGulbenkian: Lisboa, 1988.

15. van Olphen, H. E.; An Introduction to Clay Colloid Chemistry: For Clay Technologists, Geologists, and Soil Scientists, $2^{\text {nd }}$ ed., Krieger: New York, 1991.

16. Chagas, A. P.; Argilas, as essências da terra, $1^{\mathrm{a}}$ ed., Moderna: São Paulo, 1996.

17. http://www.groundwaterresearch.com.au/reference/clayLiners/clay $\% 20$ liners.htm, acessada em Março 2010.

18. Norrish, K.; Discuss. Faraday Soc. 1954, 18, 120.

19. Schubert, M.; Levine, A.; J. Am. Chem. Soc. 1955, 11, 4197.

20. Rabinovitch, E.; Epistein, L. F.; J. Am. Chem. Soc. 1970, 92, 6419.

21. Michaelis, L.; Granick, S.; J. Am. Chem. Soc. 1945, 67, 1212.

22. Bergmann, K.; O'Konski, C. T.; J. Phys. Chem. 1963, 67, 2169.

23. Neumann, M. G.; Gessner, F.; Cione, A. P. P.; Sartori, R. A.; Cavalheiro, C. C. S.; Quim. Nova 2000, 23, 818.

24. Gessner, F.; Schmitt, C. C.; Neumann, M. G.; Langmuir 1994, 10, 3749.

25. Neumann, M. G.; Gessner, F.; Schmitt, C. C.; J. Colloid Interface Sci. 1996, 177, 495

26. Gürses, A.; Karaca, S.; Dogar, Ç.; Bayrak, R.; Açıkyıldız, M.; Yalçın, M.; J. Colloid Interface Sci. 2004, 269, 310.

27. Weng, C. H.; Pan, Y. F.; J. Hazard. Mater. 2007, 144, 355.

28. Ma, Y. L.; Xu, Z. R.; Guo, T.; You, P.; J. Colloid Interface Sci. 2004, 280, 283.

29. Czímerová, A.; Jankovic, L.; Bujdák, J.; J. Colloid Interface Sci. 2004, 274, 126.

30. Jacobs, K. Y.; Schoonheydt, R. A.; Langmuir 2001, 17, 5150.

31. Bujdák, J.; Janek, M.; Madejová, J.; Komadel, P.; Clays Clay Miner. 2001, 49, 244.

32. Cenens, J.; Schoonheydt, R. A.; Clays Clay Miner. 1988, 36, 214.

33. Yuang, P. C.; Shen, Y. H.; J. Colloid Interface Sci. 2005, 285, 443.

34. van Olphen, H. E.; Fripiat, J. J.; Data Handbook for Clay Materials and other Non-Metallic Minerals, Pergamon: Oxford, 1979.

35. Bruno, T. J.; Lewandowska, A.; Tsvetkov, F.; Miller, K. E.; Hanley, H. J. M.; J. Chromatogr., A 2002, 973, 143.

36. Banin, A.; Lahav, N.; Nature 1968, 217, 1146.

37. Poli, A. P.; Batista, T.; Schmitt, C. C.; Gessner, F.; Neumann, M. G.; J. Colloid Interface Sci. 2008, 325, 386.

38. Cione, A. P. P.; Schmitt, C. C.; Neumann, M. G.; Gessner, F.; J. Colloid Interface Sci. 2000, 226, 205.

39. Neumann, M. G.; Gessner, F.; Schmitt, C. C.; Sartori, R.; J. Colloid Interface Sci. 2002, 255, 254. 